\title{
Article \\ A Comparative Analysis of Delay Propagation on Departure and Arrival Flights for a Chinese Case Study
}

\author{
Zhe Zheng ${ }^{1,2, *}$, Wenbin $\mathrm{Wei}^{3}$ and Minghua $\mathrm{Hu}{ }^{1,2}$ \\ 1 College of Civil Aviation, Nanjing University of Aeronautics and Astronautics, Nanjing 211106, China; \\ minghuahu@nuaa.edu.cn \\ 2 National Key Laboratory of Air Traffic Flow Management, Nanjing University of Aeronautics and \\ Astronautics, Nanjing 211106, China \\ 3 Department of Aviation and Technology, San Jose State University, San Jose, CA 95192, USA; \\ wenbin.wei@sjsu.edu \\ * Correspondence: zhe_zheng@outlook.com
}

Citation: Zheng, Z.; Wei, W.; Hu, M. A Comparative Analysis of Delay Propagation on Departure and Arrival Flights for a Chinese Case Study. Aerospace 2021, 8, 212. https://doi.org/10.3390/ aerospace 8080212

Academic Editor: Michael Schultz

Received: 8 June 2021

Accepted: 31 July 2021

Published: 4 August 2021

Publisher's Note: MDPI stays neutral with regard to jurisdictional claims in published maps and institutional affiliations.

Copyright: () 2021 by the authors. Licensee MDPI, Basel, Switzerland. This article is an open access article distributed under the terms and conditions of the Creative Commons Attribution (CC BY) license (https:// creativecommons.org/licenses/by/ $4.0 /)$.

\begin{abstract}
In recent years, flight delay costs the air transportation industry millions of dollars and has become a systematic problem. Understanding the behavior of flight delay is thus critical. This paper focuses on how flight delay is affected by operation-, time-, and weather-related factors. Different econometric models are developed to analyze departure and arrival delay. The results show that compared to departure delay, arrival delay is more likely to be affected by previous delays and the buffer effect. Block buffer presents a reduction effect seven times greater than turnaround buffer in terms of flight delays. Departure flights suffer more delays from convective weather than arrival flights. Convective weather at the destination airport for flight delay has a greater impact than at the original airport. In addition, sensitivity analysis of flight delays from an aircraft utilization perspective is conducted. We find that the effect of delay propagation on flight delay differs by aircraft utilization. This impact on departure delay is greater than the impact on arrival delay. In general, specific to the order of flights, the previous delay increases the impact on flight on-time performance as a flight flies a later leg. Buffer time has opposite effects on departure and arrival delay, with the order increasing. A decrease in buffer time with the order increasing, however, still has a greater reduction effect on departure delay than arrival delay. Specific to the number of flights operated by an aircraft, the more flights an aircraft flies in a day, the more the on-time performance of those flights will suffer from the previous delay and buffer time generally.
\end{abstract}

Keywords: flight delay; delay propagation; empirical analysis

\section{Introduction}

With continuous development of the world economy, flight demands have witnessed raid growth in the past decades. According to statistics from the World Bank, the number of global air travelers grew tenfold between 1970 and 2019 [1]. To improve the efficiency of the air traffic network, the air transportation sector has developed and implemented new technologies. For example, traffic flow management (TFM) has been proposed to control the overall flow of traffic in the national airspace system rather than control specific flights, compared to the traditional air traffic control system. However, the runway and airspace capacity are still not enough to manage a large number of flights. This situation has led to serious en-route and terminal traffic congestion causing rampant flight delays. According to the Joint Economic Committee report, flight delay cost the U.S. aviation industry almost $\$ 12$ billion in 2007 [2]. In China, the Civil Aviation Administration of China (CAAC) introduced a series of new regulations to prevent flight delays, such as a fine of up to 30,000 RMB when a serious flight delay occurs [3]. The European regulation EU 2019/317 is used to push air navigation service providers in Europe to perform better to improve flight on-time performance [4]. The airline industry itself has made significant 
efforts to mitigate the negative consequences of flight delays. An effective method for airlines is to adjust flight schedules to absorb delays and prevent current delays from being propagated $[5,6]$.

Considering the spatiotemporal effect of flight delays, flight departure and arrival delays are intertwined with other time components related to an aircraft. As shown in Figure 1, departure (arrival) delay is the difference between the actual departure (arrival) time and scheduled departure (arrival) time. Note that the delay mentioned in this paper refers to gate delay. The previous delay of departure delay is the arrival delay of the previous flight leg operated by the same aircraft, which is defined as "previous arrival delay", or Delay_PreArr. The delay immediately prior to the arrival delay refers to the departure delay of the same flight, which is defined as "previous departure delay" in this paper, or Delay_PreDep. During this turnaround time between the aircraft's arrival and its next departure, a series of operations are carried out, such as the unloading and loading of baggage and onboard catering supplies, the exterior inspection of the plane, and refueling if required. The time for the needed operations on the ground is called the "minimum turnaround time" or "minimum connection time" (MCT). The "turnaround buffer time", referred to as TT_dif in this paper, is defined as the difference between the scheduled turnaround time and the MCT. For flight airborne operation, the block time is calculated between the arrival and departure time of the same flight. Similar to MCT, the time for the needed operations for airborne is called minimum block time (MBT). The block buffer time is defined as the difference between the scheduled block time and the MBT, named BT_dif.

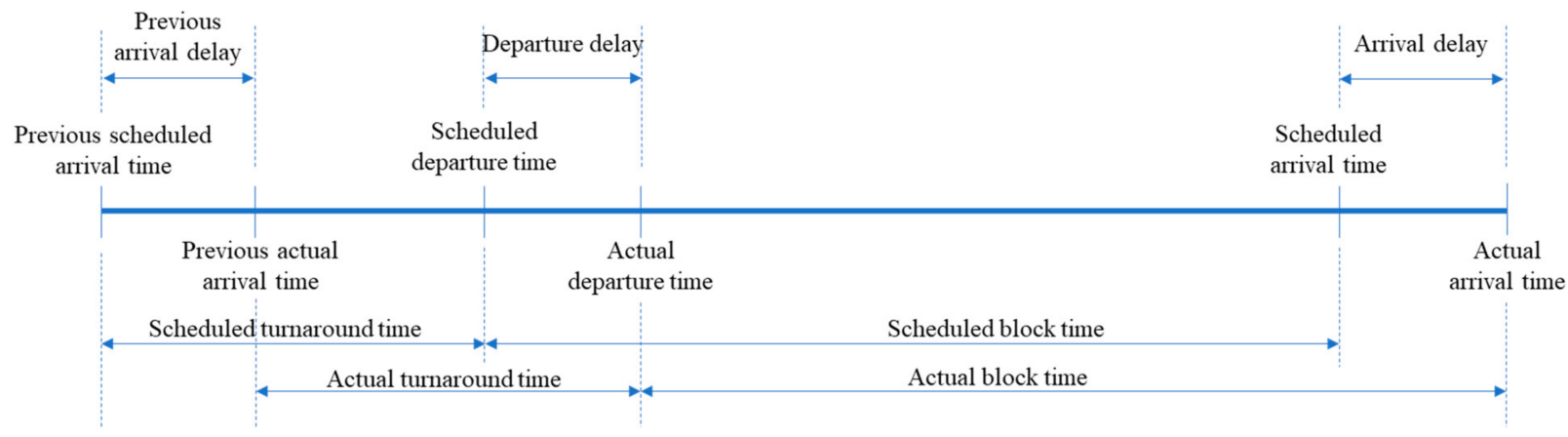

Figure 1. Time components related to a flight.

The abovementioned time components involved in a flight provide some useful perspectives on what to consider when modeling flight delay. First, as the delay propagation effect is a primary concern of the flight on-time performance in this paper, an important factor to consider is Delay_PreArr (Delay_PreDep) when analyzing the departure (arrival) delay of the current flight under study. When the difference between the actual turnaround (block) time and the scheduled one is small, departure (arrival) delay is highly correlated with Delay_PreArr (Delay_PreDep). The second important aspect is the buffer time during the ground (airborne) operation, which can account for part of the delays from the previous arrival or departure. In reality, the airline tends to insert a buffer in the scheduled time, which indicates that, except for in some extreme conditions, the actual turnaround (block) time is shorter than the scheduled time. In other words, part of Delay_PreArr (Delay_PreDep) can be absorbed when a flight is during turnaround (airborne).

In addition to delay propagation, other factors can contribute to serious delays, such as convective weather and airport congestion. Given that flight delay is susceptible to the air traffic environment, understanding the influencing factors is vitally important for improving efficiency of the air traffic system, as appropriate strategies can be implemented to alleviate the impacts of causes on flight delays both at the departure and arrival end through an efficient allocation of equipment and personnel. Therefore, the purpose of this paper is to provide effective and practical empirical models for studying flight departure 
and arrival delays considering aircraft utilization with operation-, time-, and weatherrelated variables.

This paper makes two major contributions. First, compared with US and European markets, empirical analyses on both departure and arrival delay considering the delay propagation effect of the whole Chinese market for individual flights are limited. In this paper, a comparative analysis of flight departure and arrival delay is conducted to examine how departure and arrival delay are influenced by categorizing the various factors into three groups, including the operation-, time-, and weather-related variables. Wong and Tsai [7] model both departure and arrival delay with various factors for individual flights; however, they only focus on a single OD pair of one airline. This paper provides a perspective from the air traffic network to analyze flight delay.

Second, an additional component, flight order has largely been overlooked in the existing literature. To fill this gap, the present study makes a second contribution by performing sensitivity analysis of flight delay to OrdFltOfAC (the order of the flights that an aircraft flies in a day) of a flight and NumFltOfAC (the number of the flights that an aircraft flies in a day) of an aircraft. In most cases, flight operators may try to extend buffer to make up for previous delays to improve punctuality. However, to increase productivity, it is in an airline's interest to minimize buffer in order to fly more flight legs. Therefore, such a sensitivity analysis is essential to better understand the relationship of delay propagation-related factors and flight legs.

The remainder of the paper is organized as follows. In Section 2, we present four key hypotheses, which form the basis of our model specifications. Section 3 describes the data sources and the data filtering process. Estimation results of the base model are reported and discussed in Section 4. Further sensitivity analysis of the estimation results to aircraft utilization is performed in Section 5. Findings of the paper are summarized in Section 6 along with suggestions for future research.

\section{Literature Review}

Research on flight delays has been taking place for more two decades. The majority of papers regard the delay propagation effect as an important variable. Boswell and Evans examined delay propagation that resulted from late-arriving aircraft using Markov chains and found an overall multiplier of 1.8, meaning that one minute of initial flight delay generated an additional 0.8 min of delay when propagated downstream [8]. Beatty et al. developed the concept of delay multipliers to analyze the delay propagation effect using an American Airlines crew and aircraft connectivity through an actual airline operating schedule. However, the turnaround buffer time did not account for delay propagation [9]. Later research noticed that turnaround buffer time is taken as a means to reduce propagated delays from previous flight legs. Estimation methods, including survival analysis, quantile regression, and Bayesian models, are used to analyze flight departure delay with delay propagation-related factors [10-13]. Beside the effect of delay propagation on turnaround, researchers also notice this effect when a flight is airborne. Similar to turnaround buffer time, airlines usually assert block buffer time to improve the arrival on-time performance by absorbing the departure delays. Thus, flight arrival delays tend to be shorter than delays at the departure end in general. Departure delay and airborne buffer are used as important explanatory variables to estimate or predict the arrival delay [7,14-17].

In addition to the delay propagation effect, other factors are also related to the flight delays. Baumgarten et al. explore the relationship between hubbing activities and flight delays in the United States from an airline-specific network perspective [18]. Time-related variables, such as the time of day, the day of the week, and the season, are adopted by many models to estimate flight delays. Periodic patterns are shown as significant variables in empirical models in different airports and even in different countries [16,19-21]. Air traffic flow management regulations will impact flight delays, which also increases the delay cost [22]. Flight delay is closely associated with the weather conditions at the airports of origin and destination, or en-route. Among the various causes of air flight delay, the 
weather condition has been observed as a dominant factor in many previous studies. Adverse weather, such as high surface winds, low visibility, and thunderstorms, leads to increasing takeoff or landing separations, and this further reduces the airport's capacity which is then likely to result in departure or arrival delays [23-26]. A larger part (nearly 75 percent) of system delays are related to adverse weather conditions [27]. Weatherrelated variables, such as convective weather, reduced ceiling and visibility, and wind, are presented as significant factors in the most airports [28,29]. Additionally, en-route airspace weather is an important factor. Some studies specify meteorological factors by incorporating various weather conditions to establish a Weather-Impacted Traffic Index (WITI) model. WITI data are taken as the major factors to estimate flight delays [30,31].

In view of prior research, various factors, including both delay propagation and other variables, are considered in the empirical analysis. However, seldom research compares the impact of various factors for departure and arrival delay of individual flights. Furthermore, this paper provides a different perspective of aircraft utilization when developing the flight delay models. The results can assist airlines to improve the on-time performance when scheduling the flights in the air traffic network.

\section{Model Development}

Based on the literature review and speculations in Section 1, in this section we identify several factors that may affect flight delays. Four key hypotheses about the factors are formalized in Section 3.1. Building on the hypotheses, multiple model specifications are proposed in Section 3.2 to characterize the flight-delay behavior.

\subsection{Hypotheses}

Departure (arrival) delay is the difference between the actual departure (arrival) time and the scheduled departure (arrival) time. Based on the Figure 1, the departure and arrival delay can be established as Equations (1) and (2):

Departure delay $=$ Actual departure time - Scheduled departure time,

$$
\text { Arrival delay }=\text { Actual arrival time }- \text { Scheduled arrival time. }
$$

Equations (1) and (2) are calculated from the definition of the departure and arrival delay. However, the departure and arrival delay can also be obtained from the cause of formation. Based on this, the departure and arrival delay can be obtained from Equations (3) and (4).

Departure delay $=$ Propagated arrival delay + Root departure delay,

$$
\text { Arrival delay }=\text { Propagated departure delay }+ \text { Root arrival delay }
$$

Propagated arrival delay and propagated departure delay, mentioned in Equations (3) and (4), can be further extended in Equations (5) and (6), as propagated arrival (departure) delay is impacted by Delay_PreArr (Delay_PreDep) and turnaround (block) buffer time.

Propagated arrival delay = Delay_PreArr - turnaround buffer time,

Propagated departure delay $=$ Delay_PreDep - block buffer time.

By conjecturing Equations (3) and (5), as well as (4) and (6), we obtain:

Departure delay $=$ Delay_PreArr - turnaround buffer time + Root departure delay,

$$
\text { Arrival delay }=\text { Delay_PreDep }- \text { block buffer time }+ \text { Root arrival delay. }
$$

The difference between root departure delay and turnaround buffer time reflects the difference between departure delay and previous arrival delay. Airborne flights have more priorities than flights on the ground when using the runways, considering the fuel 
limitation and safety operation. This fact indicates that the root arrival delays are more likely to be smaller than root departure delays. In addition, turnaround (block) buffer time is likely to be small, as reducing buffer time leads to more flight legs per day of an aircraft to earn more profit. Therefore, the ratio of Delay_PreDep over arrival delay may correlate more highly than the ratio of Delay_PreArr over departure delay. Therefore, the first hypothesis to be tested in the paper is formalized as follows:

Hypothesis 1 (H1). Departure (arrival) delay is relatively highly correlated with Delay_PreArr (Delay_PreDep). Everything else being equal, departure (arrival) delay should increase with Delay_PreArr (Delay_PreDep). The ratio of Delay_PreDep over arrival delay should be, on average, more than that of Delay_PreArr over departure delay.

The second hypothesis also relates to delay propagation but from a different perspective. Previous delay of a flight not only increases the operating cost of the flight but can propagate to downstream flights of the same aircraft. To mitigate delay propagation, airlines tend to set a buffer time in flight scheduled turnaround or airborne operation to absorb the previous arrival (departure) delay. If the buffer time becomes longer, then the turnaround (block) time has greater capacity to absorb and prevent delay from being propagated to the next departure or arrival. This leads to the second hypothesis of the paper.

Hypothesis 2 (H2). The ratio of departure (arrival) delay over turnaround (block) buffer time should be, on average, negative. If Hypothesis 1 can be confirmed, where the Delay_PreDep impact on arrival delay is more than Delay_PreArr impact on departure delay, the absolute value of the ratio of block buffer time over arrival delay should be, on average, more than that of turnaround buffer time over departure delay.

The third hypothesis relates to the weather condition-especially for convective weather. Convective weather inducing a large clearance or landing separation leads to a widespread airport traffic congestion. Take offs and landings are even prohibited for safety reasons in case of severe convective weather, which may cause severe flight delays. Specifically, stakeholders prefer flights to holding on the ground than holding on the airborne. Based on this policy, we propose our third hypothesis:

Hypothesis 3 (H3). Flights facing convective weather will have more restrictions to take off or land at an airport, increasing departure or arrival delay. Moreover, the departure delay is affected by convective weather more greatly than arrival delay.

Finally, our study considers that the delay propagation effect will be affected by the aircraft utilization, which includes that the order of a flight operated by an aircraft per day (OrdFltOfAC) and the number flights operated by an aircraft per day (NumFltOfAC). Considering the cumulative effect of flight delays in multi-order propagation, the latter order of a flight is, the more probability the flight on-time performance is affected by the previous flight. In addition, the more flights an aircraft flies per day, the greater the impact on downstream flights of an aircraft when delay propagation occurs. To this end, airlines tend to use a better buffer setting strategy to absorb flight delays and keep on-time performance efficiently.

Hypothesis $4 \mathbf{( H 4 ) . ~ T h e ~ a i r c r a f t ~ u t i l i z a t i o n ~ w i l l ~ i m p a c t ~ t h e ~ d e l a y ~ p r o p a g a t i o n ~ e f f e c t ~ o n ~ f l i g h t ~}$ delays. Specifically, previous delay effect grows with OrdFltOfAC increasing; buffer effect grows with NumFltOfAC increasing.

\subsection{Model Specifications}

Building on the hypotheses above, this subsection specifies econometric models for departure and arrival delay to be estimated in the paper. The general form of the departure 
and arrival delay models are presented in Equations (9) and (10) in which explanatory variables affecting departure and arrival delay are classified into three categories: operation related, time related, and weather related. The specific variables considered in the model specifications are listed in Table 1.

Departure Delay $=f($ Operation - related factors, Time - related factors, Weather - related factors $)$,

Arrival Delay $=f($ Operation - related factors, Time - related factors, Weather - related factors $)$.

Table 1. Description of variables.

\begin{tabular}{|c|c|c|}
\hline \multicolumn{2}{|c|}{ Variables } & \multirow[t]{2}{*}{ Description } \\
\hline \multicolumn{2}{|l|}{ Y-variables } & \\
\hline & DelayDep (min) & $\begin{array}{l}\text { Flight departure delay, which is the difference between actual departure time } \\
\text { and scheduled departure time }\end{array}$ \\
\hline & DelayArr (min) & $\begin{array}{l}\text { Flight arrival delay, which is the difference between actual arrival time and } \\
\text { scheduled arrival time }\end{array}$ \\
\hline \multicolumn{3}{|l|}{ X-variables } \\
\hline \multirow{9}{*}{ Operation related } & Delay_PreArr (min) & Arrival delay of the previous flight of the same aircraft \\
\hline & Delay_PreDep (min) & Departure delay of the current flight \\
\hline & TT_dif (min) & Difference of the scheduled turnaround time and minimum turnaround time \\
\hline & BT_dif (min) & Difference of the scheduled block time and minimum block time \\
\hline & CumDepDelay (min) & Cumulated departure delay in the past hour of the airport under study \\
\hline & CumArrDelay (min) & Cumulated arrival delay in the past hour of the airport under study \\
\hline & Widebody & Takes value 1 if a flight is associated with a wide body aircraft \\
\hline & HubDep & Takes value 1 if the flight departs from a hub airport \\
\hline & HubArr & Takes value 1 if the flight arrives at a hub airport \\
\hline \multirow{5}{*}{ Time related } & DT & $\begin{array}{l}\text { Takes value } 1 \text { if the flight operates in the day (scheduled departure between } \\
\text { 6:00 and 20:00), and } 0 \text { otherwise }\end{array}$ \\
\hline & Wkd & Takes value 1 if the flight operates on a weekday, and 0 otherwise \\
\hline & Sprg & Takes value 1 if the flight is scheduled to depart in Spring, and 0 otherwise \\
\hline & Sumr & Takes value 1 if the flight is scheduled to depart in Summer, and 0 otherwise \\
\hline & Fall & Takes value 1 if the flight is scheduled to depart in Fall, and 0 otherwise \\
\hline \multirow{8}{*}{ Weather related } & ConvecWthrDep & Takes value 1 if a flight is in the convective weather at the departure airport \\
\hline & ConvecWthrArr & Takes value 1 if a flight is in the convective weather at the arrival airport \\
\hline & TempDepDif (Celsius) & $\begin{array}{l}\text { Absolute difference between the temperature at the actual departure time of a } \\
\text { flight and the average temperature associated with all departure flights of the } \\
\text { whole year at the same airport }\end{array}$ \\
\hline & TempArrDif (Celsius) & $\begin{array}{l}\text { Absolute difference between the temperature at the actual arrival time of a } \\
\text { flight and the average temperature associated with all arrival flights of the } \\
\text { whole year at the same airport }\end{array}$ \\
\hline & $\begin{array}{l}\text { WdspDep (meter per } \\
\text { second) }\end{array}$ & $\begin{array}{l}\text { Wind speed during the actual departure hour of a flight at the corresponding } \\
\text { airport }\end{array}$ \\
\hline & $\begin{array}{l}\text { WdspArr (meter per } \\
\text { second) }\end{array}$ & $\begin{array}{l}\text { Wind speed during the actual arrival hour of a flight at the corresponding } \\
\text { airport }\end{array}$ \\
\hline & VisDep (kilometers) & $\begin{array}{l}\text { Log of visibility (in miles) plus one mile during the actual departure hour at } \\
\text { the departure airport }\end{array}$ \\
\hline & VisArr (kilometers) & $\begin{array}{l}\text { Log of visibility (in miles) plus one mile during the actual arrival hour at the } \\
\text { arrival airport }\end{array}$ \\
\hline
\end{tabular}


To understand the impact of various factors on both departure and arrival delays, two separate specifications are considered: one for departure delay, termed the DelayDep model, as shown in Equation (9); the other for arrival delay, termed the DelayArr model, as shown in Equation (10).

Operation-related factors include the previous delay, buffer time, airport congestion, aircraft type, and hubs. For different explanatory variables of DelayDep and DelayArr model, some variables, including the previous delay, buffer time, and airport congestion, have different definitions in two models. Considering the different operational behaviors at departure and arrival, three factors, including the previous delay, buffer time, and airport congestion, have different definitions in the DelayDep and DelayArr models. For departure delay, the three factors are defined as previous arrival delay, turnaround buffer time, and cumulated departure delays in an airport. However, to analyze the arrival delay, the three factors are defined as previous departure delay, airborne buffer time, and cumulated arrival delays in an airport.

For operation-related variables, one variable is previous delay, which is intended to capture the delay propagation effect from the previous departure or arrival. In addition, propagated delay is one of the most important parts of the flight delays [17] (Tan et al., 2021). The longer the previous delay is, the more likely the on-time performance of the flight under study will be affected by the previous delay. In the DelayDep model, the previous delay refers to Delay_PreArr. In the DelayArr model, the previous delay refers to Delay_PreDep. This variable is used to test Hypothesis 1.

The variables TT_dif and BT_dif are constructed to test Hypothesis 2 for DelayDep and DelayArr model, respectively. TT_dif (BT_dif) is defined as the difference between the scheduled turnaround (block) time and MCT (MBT). To calculate MCT, CAAC documents MCT values but does not provide details on how the values are obtained [32]. Another method applies the statistical method to regard the 5 th percentile observed turnaround time follows the idea of existing work, which makes the calculation more robust to measurement error and reduces the influence of unusually favorable conditions $[15,33,34]$. In this paper, we prefer to use the latter method by regarding the 5th percentile observed turnaround time as MCT. Similarly, the MBT calculation also follows this statistical method. The expectation is that the less buffer time there is, the more difficult it is to maintain the scheduled departure or arrival times of flights with large previous delays.

In this paper, we use the CumDepDelay for DelayDep model and CumArrDelay for DelayArr model to present the departure and arrival congestion, respectively [11]. CumDepDelay and CumArrDelay respectively measure the departure and arrival delays generated in the airports during the past hour. All the flight records departing from (arriving at) the airports are used to calculate the CumDepDelay (CumArrDelay), if the exact the time information (scheduled departure time, scheduled arrival time, actual departure time, and actual arrival time) can be obtained. The expectation is that serious airport delays may increase the flight delays. We use HubDep and HubArr to indicate whether a flight takes off from or lands at the airline's hub. We speculate that a flight from or to a hub airport is likely to receive better ATC services in the terminal airspace. In addition, an airline usually has more flying experience and a stronger relationship with ATC service providers at its hub airports, which may improve flight operations.

The DelayDep and DelayArr models share a number of common time and weatherrelated variables. Several time variables are considered in this paper. The first one is DT, a dummy variable indicating whether a flight operates in the day or night. DT is set to be 1 if the scheduled departure (arrival) time is between 6:00 and 20:00. We postulate that there are more flights in the daytime than in the night, which will lead to traffic congestion. Second, flight delays may vary depending on whether it is a weekday or the weekend, again considering the difference in air traffic, particularly for business travel. A Wkd variable is included, which takes the value of 1 if the scheduled departure day is a weekday and 0 otherwise. Third, seasonal changes also affect the traffic demand and thus impact airline operation strategies, which may further affect flight delays. To this end, 
three seasonable variables, including Sprg, Sumr, and Fall, are introduced as explanatory variables.

To capture the impacts of weather on flight delays, we consider convective weather, temperature, wind speed, and visibility at the departure and arrival airports of a flight. The convective weather (ConvecWthrDep and ConvecWthrArr) is the key variable among the weather-related factors, measuring whether there is a thunderstorm at the departure or arrival end. A thunderstorm can affect an aircraft's operation safety, further leading to severe delays. For temperature, we assume that both hot and cold temperatures present adverse weather and impact the flight delays. Hot temperature negatively affects aircraft engine performance [35], whereas cold temperature is often associated with foggy and snowy days, which may cause poor performance on the airport surface and thereby also negatively affect flight delays. The absolute difference between the temperature at the scheduled departure (arrival) time of a flight and the average temperature associated with all departure (arrival) flights of the whole year at the same airport are considered (TempDepDif and TempArrDif). The wind-speed variables (WdspDep and WdspArr) provide the speed of the wind at the departure (arrival) airport during the hour of the scheduled departure (arrival) time of the flight. The visibility variables (VisDep and VisArr) are similarly defined but taking logarithmic values to capture the plausible nonlinear relationship between visibility and flight delay. For example, visibility decreasing from $1 \mathrm{~km}$ to $0.5 \mathrm{~km}$ impacts flight operations more than decreasing from $8 \mathrm{~km}$ to $7.5 \mathrm{~km}$. As some records have visibility of zero miles, we add one to the exponent: VisDep $=\ln ($ Departure Visibility +1$)$ and VisArr $=\ln ($ Arrival Visibility +1$)$. Eight weather variables, including ConvecWthrDep, ConvecWthrArr, TempDepDif, TempArrDif, WdspDep, WdspArr, VisDep, and VisArr, are all considered in departure and arrival delays. This is because the departure flights will be delayed if the weather condition at the arrival airport is not appropriate for landing. The weather condition at the departure airport may extend the taxiing time or even keep waiting until aircraft enters the runway to further impact arrival delays.

Following the discussions, below are the complete DelayDep and DelayArr model specifications. In the specifications, $\alpha^{\prime}$ s and $\beta^{\prime}$ s are the coefficients to be estimated, and $\varepsilon$ and $v$ are the error terms.

$$
\begin{gathered}
\text { DelayDep }=\alpha_{0}+\alpha_{1} \text { Delay_PreArr }+\alpha_{2} \text { TT_dif }+\alpha_{3} \text { CumDepDelay }+\alpha_{4} \text { Widebody }+\alpha_{5} \text { HubDep } \\
+\alpha_{6} \text { HubArr }+\alpha_{7} \text { DT }+\alpha_{8} \text { Wkd }+\alpha_{9} \text { ConvecWthrDep }+\alpha_{10} \text { ConvecWthrArr } \\
+\alpha_{11} \text { TempDepDif }+\alpha_{12} \text { TempArrDif }+\alpha_{13} \text { WdspDep }+\alpha_{14} \text { WdspArr }+\alpha_{15} \text { VisDep }+\alpha_{16} \text { VisArr }+\varepsilon,
\end{gathered}
$$

$$
\begin{gathered}
\text { DelayArr }=\beta_{0}+\beta_{1} \text { Delay_PreDep }+\beta_{2} \text { BT_dif }+\beta_{3} \text { CumArrDelay }+\beta_{4} \text { Widebody }+\beta_{5} \text { HubDep } \\
+\beta_{6} \text { HubArr }+\beta_{7} \text { DT }+\alpha_{8} \text { Wkd }+\beta_{9} \text { ConvecWthrDep }+\beta_{10} \text { ConvecWthrArr } \\
+\beta_{11} \text { TempDepDif }+\beta_{12} \text { TempArrDif }+\beta_{13} \text { WdspDep }+\beta_{14} \text { WdspArr }+\beta_{15} \text { VisDep } \\
+\beta_{17} \text { VisArr }+v .
\end{gathered}
$$

\section{Data}

\subsection{Data Source}

The data needed for model estimation come from several sources. For operation- and time-related variables, flight-level records are collected for the year 2016 from the Air Traffic Management Bureau of CAAC. This results in the collection of 3,705,093 flight records in China, including commercial and general aviation flights. Each record documents the departure and arrival airports, departure and arrival dates, airline to which the flight belongs, aircraft type of the flight, tail number of the aircraft, the scheduled departure time, the actual departure time, the scheduled arrival time, and the actual arrival time. The time information allows us to construct DelayDep, DelayArr, Delay_PreDep, Delay_PreArr, TT_dif, BT_dif, CumDepDelay, CumArrDelay, DT, Wkd, and the seasonal dummy variables. For weather-related variables, the Meteorological Terminal Aviation Routine (METAR) weather reports of top 30 airports provide weather information including temperature, 
wind speed, visibility, and convective weather at each airport covered in the dataset at 60-min intervals.

\subsection{Data Filtering}

Two data filtering steps are performed to ensure data consistency. First, flight records with missing variable values are eliminated from the data. For instance, flights for which the actual arrival time is empty in the dataset are treated as cancelled flights and have been omitted from this paper. Second, as the original dataset is not collected completely, several crucial items in the dataset - especially for actual departure time, actual arrival time, aircraft type, and aircraft tail number-are abnormal or incorrect, leading to erroneous calculations of explained or explanatory variables. Such records have been omitted.

In addition to the above, insofar as we are interested in flight delays of commercial flights of Chinese airlines, other flights have been removed. Furthermore, we focus on flights between airports that have high levels of traffic and are thus subject to congestion. In this study, we focus on flights that flew between the top 30 airports in terms of total flight operations in 2016. These airports are shown in Appendix A and are also listed in Appendix A. This process reduces the number of flight records to 1,469,909 for modeling. However, we notice that there are some flight records with delays (both for departure and arrival) earlier than a day or some records with negative buffer time (both for turnaround and block buffer). These records are also regarded as abnormal records and deleted from the dataset. When analyzing the departure delays, 1,426,688 records are retained. As for studying arrival delays, 1,459,634 records are retained. The summary statistics for some of the variables are reported in Table 2.

Table 2. Summary statistics of the variables in the specified models after data cleaning.

\begin{tabular}{cccccc}
\hline Variable & Flight Phase & Mean & Std. Dev. & Min. & Max. \\
\hline \multirow{2}{*}{ Delay * } & Departure & 13.2 & 53.2 & -1432 & 1407 \\
& Arrival & 15.3 & 52.9 & -1437 & 1410 \\
\hline \multirow{2}{*}{ Previous } & Departure & 9.1 & 38.8 & -883 & 1408 \\
delay ${ }^{*}$ & Arrival & 14.2 & 54.4 & -1432 & 1407 \\
\multirow{2}{*}{ Buffer * } & Departure & 269.7 & 391.6 & 0 & 9444 \\
& Arrival & 344.8 & 473.0 & 0 & 11,646 \\
\hline \multirow{2}{*}{ CumDelay * } & Departure & 24.3 & 50.2 & 0 & 462.3 \\
& Arrival & 4.8 & 5.3 & 0 & 1345.0 \\
\hline \multirow{2}{*}{ TempDepDif } & Departure & 8.7 & 6.3 & 0.2 & 52.2 \\
& Arrival & 8.7 & 6.2 & 0.4 & 50.4 \\
\hline \multirow{2}{*}{ TempArrDif } & Departure & 8.7 & 6.3 & 0.3 & 52.3 \\
& Arrival & 8.7 & 6.2 & 0.3 & 51.3 \\
\hline \multirow{2}{*}{ WdspDep } & Departure & 3.4 & 2.1 & 0.0 & 18.0 \\
& Arrival & 3.4 & 2.1 & 0.0 & 22.0 \\
\hline \multirow{2}{*}{ WdspArr } & Departure & 3.4 & 2.1 & 0.0 & 17.0 \\
& Arrival & 3.4 & 2.1 & 0.0 & 17.0 \\
\hline \multirow{2}{*}{ VisDep } & Departure & 2.1 & 0.4 & 0 & 2.4 \\
& Arrival & 2.1 & 0.4 & 0 & 2.4 \\
\hline \multirow{2}{*}{ VisArr } & Departure & 2.1 & 0.4 & 0 & 2.4 \\
& Arrival & 2.1 & 0.4 & 0 & 2.4 \\
\hline
\end{tabular}

* Delay for Departure refers to DelayDep, Delay for Arrival refers to DelayArr; ${ }^{*}$ Previous delay for Departure refers to DelayDep, Previous delay for Arrival refers to DelayArr; * Buffer for Departure refers to TT_dif, Buffer for Arrival refers to BT_dif; ${ }^{*}$ CumDelay for Departure refers to CumDepDelay, CumDelay for Arrival refers to CumArrDelay. 


\section{Estimation Results}

This section presents the estimation results of the DelayDep and DelayArr models specified in Section 2. To analyze the marginal effect of each variable, we estimate the DelayDep and DelayArr models using ordinary least square (OLS) with clustered standard error departure airports and arrivals, respectively, which is more efficient than to use conventional standard errors when heteroskedasticity is present [36].

The estimation results are presented in Table 3. Looking at the individual coefficients, the previous delay coefficients are 0.698 of Delay_PreArr and 0.826 of Delay_PreDep for the departure delay and arrival delay, which matches Hypothesis 1's speculation that the previous delay, including Delay_PreArr and Delay_PreDep, would present positive effects and relatively high correlation with departure and arrival delay, respectively. The marginal effect of arrival delay over Delay_PreDep is more than that of departure delay over Delay_PreArr. We also observe that the coefficient of Delay_PreDep is more than that of Delay_PreArr, implying that the flight arrival delay is more likely to be impacted by the propagated effects. The next variable of interest is the buffer effect, including TT_dif in the DelayDep model and BT_dif in the DelayArr model, which presents the propagation effect from different perspectives with the previous delay. Both TT_dif and BT_dif indicate negative signs for departure and arrival delay, respectively, and thus confirm Hypothesis 2, that flights with larger buffer times will have more capacity to reduce flight delays. Specifically, we observe that the BT_dif has a much larger reduction (0.744 $\mathrm{min})$ for flight delays_-about nine times greater-than the reduction of TT_dif $(0.082$ $\mathrm{min}$ ). Therefore, inserting buffer time when the aircraft is airborne can obtain the flight on-time performance more efficiently and also help airlines save time for operating more flight legs.

Table 3. Base models for flight delays for both departure and arrival.

\begin{tabular}{|c|c|c|c|c|}
\hline \multirow[b]{2}{*}{ Variable } & \multicolumn{2}{|c|}{ Departure } & \multicolumn{2}{|c|}{ Arrival } \\
\hline & Est. & Std. Err. & Est. & Std. Err. \\
\hline Delay_PreArr & $0.698^{* * *}$ & 0.016 & & \\
\hline Delay_PreDep & & & $0.826^{* * *}$ & 0.009 \\
\hline TT_dif & $-0.082 * * *$ & 0.006 & & \\
\hline BT_dif & & & $-0.744^{* * *}$ & 0.025 \\
\hline CumDepDelay & $0.033^{* * *}$ & 0.003 & & \\
\hline CumArrDelay & & & $0.009^{* * *}$ & 0.001 \\
\hline Widebody & $6.908 * * *$ & 1.795 & -0.973 & 0.909 \\
\hline HubDep & $-5.699 * * *$ & 1.535 & 0.197 & 0.433 \\
\hline HubArr & 0.884 * & 0.481 & 0.129 & 0.814 \\
\hline Daytime & 0.559 & 0.630 & $2.234^{* * *}$ & 0.288 \\
\hline Weekday & $0.397^{* * *}$ & 0.123 & $0.626^{* * *}$ & 0.074 \\
\hline Sprg & -0.551 & 0.480 & $-1.143^{* * *}$ & 0.377 \\
\hline Sumr & $1.950 * * *$ & 0.579 & $-0.626^{*}$ & 0.357 \\
\hline Fall & $1.681^{* * *}$ & 0.358 & $-1.204^{* *}$ & 0.461 \\
\hline ConvecWthrDep & $16.818^{* * *}$ & 2.813 & $9.232^{* * *}$ & 0.448 \\
\hline ConvecWthrArr & $21.817^{* * *}$ & 1.433 & $13.967^{* * *}$ & 1.126 \\
\hline TempDepDif & 0.014 & 0.047 & $0.134^{* * *}$ & 0.019 \\
\hline TempArrDif & 0.013 & 0.035 & 0.001 & 0.039 \\
\hline WdspDep & $0.423^{* * *}$ & 0.107 & 0.014 & 0.049 \\
\hline WdspArr & $0.475 * * *$ & 0.068 & $0.410^{* * *}$ & 0.105 \\
\hline VisDep & $-1.892 * * *$ & 0.816 & $-1.857^{* * *}$ & 0.259 \\
\hline VisArr & $-3.401^{* *}$ & 0.340 & $-0.980^{* *}$ & 0.417 \\
\hline Cons & $7.076^{* * *}$ & 1.591 & $5.867^{* * *}$ & 0.808 \\
\hline$R^{2}$ & & & & \\
\hline Num. Obs. & & & & \\
\hline
\end{tabular}


For the remaining operation-related variables, the CumDepDelay (CumArrDelay) variable has a significant coefficient in the DelayDep (DelayArr) model. In particular, the coefficient of CumDepDelay is larger than that of CumArrDelay, which indicates that arrival delay is less affected by airport congestion than departure delay. A probable explanation is that arrival flights have priority in using the runway, when airport congestion exists. In reality, ATC controllers prefer to have flights waiting on the ground, because the airborne holding time for arrival flights is limited, considering the fuel supply the aircraft must have to safety stay in flight. Aircraft type, simplified into wide body and non-wide body aircraft in this paper, is positive, with a larger magnitude (almost seven minutes) in departure delay as opposed to a negative but smaller (less than one minute) and insignificant for the arrival delay, which indicates that aircraft type plays an important role in departure delays. A possible explanation is that the wide body aircraft has stricter conditions to take off from the runway [37].

For the hub airport variables, the negative coefficients of the departure model reveal that using a hub airport either at the departure or arrival will help reduce departure delays. The HubDep effect is larger than the HubArr effect in the departure model, with 5.7-min decrease, which is the most significant reduction to occur at the departure end. This is reasonable as such flights may receive better ATC support near the terminal airspace. However, for arrival delays, the hub factors present tiny impacts; even the arrival hub presents an insignificant coefficient. In fact, except that the departure hub for the departure model shows a notable effect, either hub factor for the arrival model or the arrival hub for the departure model has tiny effect in terms of delays. Facing of a complex operation environment at the destination airport, the landing aircraft has more priority than the take-off aircraft, as waiting for a long time in the airborne will increase the operational risk both for aircraft and terminal airspace.

For the time-related variables, DT takes positive values for departure and arrival delays, with the coefficient for the departure model presenting insignificantly. It indicates that, on average, departure flights in the daytime will generate delays less than arrival flights. Positive coefficients in both models indicate that larger traffic demands during weekdays also lead to greater delays both for departure and arrival flights. With regard to seasonal effects, the departure model reveals that flights generally have the longest departure delays in the summer and the longest arrival delays in the winter. Seasonal effects are stronger for departure flights, with a 2.5-min difference between the "longest" and the "shortest" seasons, while for arrival delays, this difference is only $1.2 \mathrm{~min}$.

Turning to the weather-related variables, the variables of departure delay have more impact than those for arrival delays. This indicates that aircraft on the ground are more likely to keep waiting on a better weather condition than airborne aircraft, mainly to ensure flight safety. Convective weather variables (ConvecWthrDep and ConvecWthrArr) are the two dominant coefficients among all dummy variables. Furthermore, the influence of convective weather variables is much higher than the rest of the weather-related factors, even if the rest factors reach the severe condition. Convective weather presents positive coefficients significantly both at the origin and destination airports, while convective weather at the destination airport plays a more important role for delays. Moreover, convective weather impacts departure flights more than arrival flights. This matches Hypothesis 3 that the convective weather will affect the flight on-time performance greatly, and departure delay is more susceptible than arrival delay. Temperature and wind speed at the airport surface generally present small effects. For departure delays, temperature at the origin and destination airports present positive effects. However, for arrival delays, only temperature at departure airports is impactful. Both departure and arrival delays increase as the wind speed increases. With the exception of convective weather, visibility is the most important factor affecting both departure and arrival delays; better visibility helps decrease separation while forming the take-off sequence or landing queue, which contributes to reducing delays. 


\section{Sensitivity Analysis to Aircraft Utilization}

Aircraft fly to numerous airports sequentially according to their flight plans. Considering the spatiotemporal effect of delay propagation in the air traffic network, this section investigates the sensitivity of the estimation results to aircraft utilization (OrdFltOfAC and NumFltOfAC). OrdFltOfAC focuses on the sequence of flights operated by an aircraft; NumFltOfAC considers the number of flights operated by an aircraft within a day.

\subsection{Sensitivity to the Order of Flight Leg}

Flights tend to present different on-time performances in different orders of flight leg. In the early orders of the flights per day, flights are less likely to be affected by previous legs, especially for the flight of the first order with no delay propagation impact. In contrast, in the late orders of an aircraft, flight on-time performance is likely to be affected by many previous flight legs. Considering that flights in different orders may show the different delay propagation effects, the buffers during the turnaround and en-route also affect the difference in flight delays. Figure 2 illustrates the distribution of flights for departures and arrivals in seven groups based on OrdFltOfAC: 1, 2, 3, 4, 5, 6, and >6. Obviously, as the OrdFltOfAC increases, the number of flights decreases. Both for departures and arrivals, the flights of the first four order accounted for nearly 90 percent of the total flights, which indicates that there are few flights above the fourth order. Flights with the first order accounted for nearly thirty percent of the total flights among the top 30 airports, indicating that nearly thirty percent of the flights are not affected by the previous arrival delay at the departure ends.

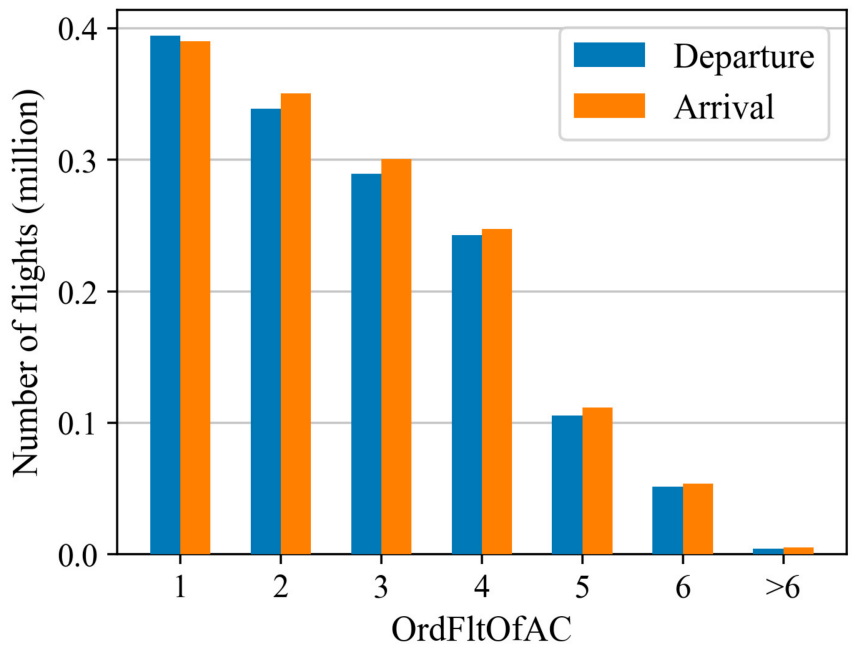

Figure 2. Distribution of the order of flight legs flown by aircraft in China.

Table 4 reports the estimation results for each of the seven flight groups for both departure and arrival delays. All standard errors in the departure and arrival models are clustered by departure and arrival airports, respectively. In this subsection, we focus on the coefficients for previous delay (Delay_PreArr in the departure model and Delay_PreDep in the arrival model) and buffer effect (TT_dif in the departure model and BT_dif in the arrival model), where all of the point estimates and $95 \%$ confidence intervals are plotted in Figure 3. In Figure 3a, the coefficients of both Delay_PreArr and Delay_PreDep follow an increasing trend matching Hypothesis 4, and the increase is more prominent in departure delays. This indicates that the latter order of an aircraft in a day suffer a heavy delay, which is propagated by the previous arrival (departure). One possible explanation is that the on-time performance of the latter-order flight can be affected by more than one previous flight leg. For example, if the delays generated in the second order, which are not fully absorbed in the third order, this will continue to affect the on-time performance of the fourth order. Moreover, arrival delays are more likely to be impacted by the previous delay 
(the coefficients of Delay_PreDep are larger than that of Delay_PreArr). When OrdFltOfAC is above two, the coefficients of Delay_PreDep in arrival model are all greater than 0.9. This indicates that all the departure delays are almost propagated to the arrival delays. The impacts of Delay_PreArr and Delay_PreDep on flight delays reach the maximum while OrdFltOfAC is above six (up to 0.866 when modeling departure delay, up to 0.985 when modeling arrival delay). This indicates that all the departure delays are almost propagated to the arrival delays.

Table 4. Estimation results of flight delays for departure and arrival in different OrdFltOfAC.

\begin{tabular}{|c|c|c|c|c|c|c|c|}
\hline Departure & 1 & 2 & 3 & 4 & 5 & 6 & $>6$ \\
\hline Delay_PreArr & 0 & $0.642 * * *$ & $0.653 * * *$ & $0.782^{* * *}$ & $0.711^{* * *}$ & $0.813^{* * *}$ & $0.866^{* * *}$ \\
\hline TT_dif & 0 & $-0.084 * * *$ & $-0.106^{* * *}$ & $-0.140 * * *$ & $-0.123 * * *$ & $-0.191 * * *$ & $-0.147^{* * *}$ \\
\hline CumDepDelay & $0.037 * * *$ & $0.036^{* *}$ & $0.033^{* * *}$ & $0.026^{* * *}$ & $0.026^{* * *}$ & $0.018^{* * *}$ & $0.013^{* * *}$ \\
\hline Widebody & $9.284^{* * *}$ & $5.307^{* * *}$ & 3.236 * & $7.000 * * *$ & $6.714^{* *}$ & -0.657 & 0 \\
\hline HubDep & $-6.028 * *$ & $-4.924^{* * *}$ & $-3.899 * * *$ & $-4.622 * * *$ & $-3.133 * *$ & $-4.942 * * *$ & $-8.085^{* *}$ \\
\hline HubArr & -0.123 & 0.331 & $1.509 * *$ & $1.216^{* *}$ & $2.484 * *$ & 1.548 & -0.389 \\
\hline Daytime & $-33.425^{* * *}$ & $5.038 * * *$ & 1.514 & 0.821 & 1.582 & $2.888 * *$ & 0.142 \\
\hline Weekday & $0.677^{* *}$ & $0.509 * *$ & $0.549 * *$ & 0.216 & $-0.515 *$ & -0.053 & 1.209 \\
\hline Sprg & $1.652 *$ & $-1.145 * *$ & $-1.769^{* * *}$ & -0.938 & -0.868 & -1.172 & -0.766 \\
\hline Sumr & $3.208^{* * *}$ & $2.613^{* * *}$ & 1.264 & $1.954^{* *}$ & $1.913^{* * *}$ & 0.783 & -0.337 \\
\hline Fall & $2.065^{* * *}$ & $1.867 * * *$ & $2.258^{* * *}$ & $2.546^{* * *}$ & $2.291 * * *$ & 0.108 & -0.911 \\
\hline ConvecWthrDep & $13.225 * * *$ & $18.063^{* * *}$ & $18.212 * * *$ & $17.448^{* * *}$ & $18.151 * * *$ & $15.808^{* * *}$ & $22.173^{* * *}$ \\
\hline ConvecWthrArr & $15.319^{* * *}$ & $28.221 * * *$ & $23.924 * * *$ & $20.517 * * *$ & $18.674^{* * *}$ & $18.457^{* * *}$ & $12.325 * *$ \\
\hline TempDepDif & 0.004 & 0.025 & -0.001 & 0.016 & -0.045 & -0.019 & 0.102 \\
\hline TempArrDif & 0.064 & -0.025 & 0.008 & $-0.085^{* *}$ & -0.011 & -0.016 & $-0.306^{* *}$ \\
\hline WdspDep & 0.568 ** & $0.233^{* *}$ & 0.165 & 0.178 & -0.059 & 0.124 & 0.416 \\
\hline WdspArr & $0.546 * * *$ & $0.287^{* * *}$ & $0.357^{* * *}$ & 0.097 & 0.089 & -0.201 & $-0.600^{* *}$ \\
\hline VisDep & $-4.578 * * *$ & $-1.331 *$ & -1.287 & -0.76 & $-1.683 * *$ & -0.17 & 1.537 \\
\hline VisArr & $-6.267 * * *$ & $-3.897^{* * *}$ & $-2.768^{* * *}$ & $-1.964 * * *$ & $-1.833^{* * *}$ & $-1.096 * *$ & 2.083 \\
\hline Cons & $45.059 * * *$ & $7.262 * * *$ & $8.191^{* * *}$ & $6.312 * * *$ & $8.480^{* * *}$ & $3.478 *$ & -6.508 \\
\hline Num. Obs. & 394,275 & 338,801 & 289,459 & 242,664 & 105,433 & 51,612 & 4444 \\
\hline$R^{2}$ & 0.083 & 0.426 & 0.468 & 0.604 & 0.551 & 0.650 & 0.630 \\
\hline Arrival & 1 & 2 & 3 & 4 & 5 & 6 & $>6$ \\
\hline Delay_PreDep & $0.596^{* * *}$ & $0.904^{* * *}$ & $0.906^{* * *}$ & $0.946^{* * *}$ & $0.958^{* * *}$ & $0.943^{* * *}$ & $0.985^{* * *}$ \\
\hline BT_dif & $-0.743 * * *$ & $-0.752 * * *$ & $-0.741^{* * *}$ & $-0.712 * * *$ & $-0.742 * * *$ & $-0.674 * * *$ & $-0.605 * * *$ \\
\hline CumArrDelay & $0.020 * * *$ & $0.008^{* * *}$ & $0.006^{* * *}$ & $0.005^{* * *}$ & $0.004^{* * *}$ & $0.003^{* * *}$ & 0 \\
\hline Widebody & -1.169 & $-2.564 * * *$ & $-1.944 * * *$ & -1.317 & 1.374 & -1.603 & $-28.534^{* * *}$ \\
\hline HubDep & $1.904 * *$ & -0.431 & $-1.887 * * *$ & $-1.099 *$ & $-1.817^{* *}$ & -0.872 & $-4.596 * *$ \\
\hline HubArr & -0.503 & 0.007 & 0.31 & 0.613 & 0.529 & -0.143 & 0.189 \\
\hline Daytime & $-6.322 * * *$ & $-2.851 * * *$ & -0.068 & $2.168^{* * *}$ & $1.556^{* * *}$ & $4.958^{* *}$ & $3.925 * *$ \\
\hline Weekday & $0.810^{* * *}$ & $0.481 * * *$ & $0.371^{* * *}$ & $0.166 *$ & $0.402 * * *$ & 0.284 * & 0.776 \\
\hline Sprg & $-2.701 * * *$ & -0.065 & $-0.787^{*}$ & -0.201 & 0.406 & -0.1 & -0.244 \\
\hline Sumr & $-1.111^{* * *}$ & $-0.819 *$ & -0.636 & $-0.867 *$ & $\begin{array}{l}0.400 \\
-0.302\end{array}$ & -0.875 & $\begin{array}{l}-0.244 \\
0.261\end{array}$ \\
\hline Fall & $-1.405^{* *}$ & $-1.960 * * *$ & $-1.048 * *$ & $-1.319 * *$ & $-0.877^{*}$ & -0.518 & 0.281 \\
\hline ConvecWthrDep & $14.856^{* * *}$ & $6.222 * * *$ & $7.266^{* * *}$ & $7.566^{* * *}$ & $10.857^{* * *}$ & $6.071^{* * *}$ & $6.015^{* *}$ \\
\hline ConvecWthrArr & $17.781 * * *$ & $14.357 * * *$ & $11.001 * * *$ & $12.176^{* * *}$ & $12.891 * * *$ & $10.455 * * *$ & $13.680 * * *$ \\
\hline TempDepDif & $0.232 * * *$ & $0.081^{* * *}$ & $0.156^{* * *}$ & $0.093^{* * *}$ & 0.045 & 0.049 & -0.02 \\
\hline TempArrDif & 0.032 & 0.026 & -0.044 & 0.007 & -0.026 & -0.028 & 0.056 \\
\hline WdspDep & $0.203^{* * *}$ & $-0.114 *$ & $-0.129^{* *}$ & $-0.132 * *$ & 0.03 & 0.032 & 0.053 \\
\hline WdspArr & $0.480 * * *$ & $0.354 * * *$ & $0.322 * * *$ & $0.487 * * *$ & $0.454 * * *$ & $0.434 * * *$ & $0.539 *$ \\
\hline VisDep & $-3.969 * * *$ & $-1.387^{* * *}$ & $-1.435^{* * *}$ & $-1.044^{* * *}$ & $-0.960 * * *$ & $-0.806 * *$ & 1.059 \\
\hline VisArr & $-1.347^{* *}$ & $-0.844^{* *}$ & $-0.904^{* *}$ & -0.72 & $-0.889^{*}$ & 0.239 & -0.583 \\
\hline Cons & $17.441^{* * *}$ & $9.065^{* * *}$ & $7.667^{* * *}$ & $2.880^{* *}$ & $3.944^{* * *}$ & 0.067 & $-3.476^{*}$ \\
\hline Num. Obs. & 390,292 & 350,531 & 300,773 & 247,605 & 111,705 & 53,761 & 4967 \\
\hline$R^{2}$ & 0.551 & 0.825 & 0.848 & 0.894 & 0.900 & 0.900 & 0.961 \\
\hline
\end{tabular}

Figure $3 b$ shows the Buffer coefficient estimates in different OrdFltOfAC, revealing that the changing trends of buffer coefficient for departure and arrival models are in opposite directions. While the Buffer effect increases with OrdFltOfAC in the departure models, it presents a decreasing trend for arrival on-time performance. Nonetheless, block buffer (BT_dif) presents a better delay absorption than turnaround buffer (TT_dif) in any OrdFltOfAC. This inspires airlines to insert buffers when aircraft are airborne, which increases the efficiency to reduce flight delays. In the first order of flight legs, the difference in the Buffer coefficient between the departure and arrival models is nearly 0.75 . However, after sixth flight order, the difference in buffer effect between two models is less than 0.45. 


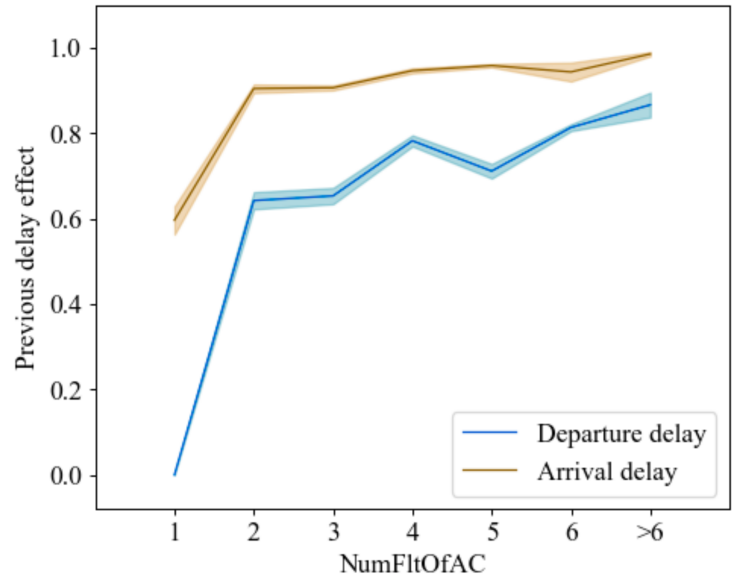

(a)

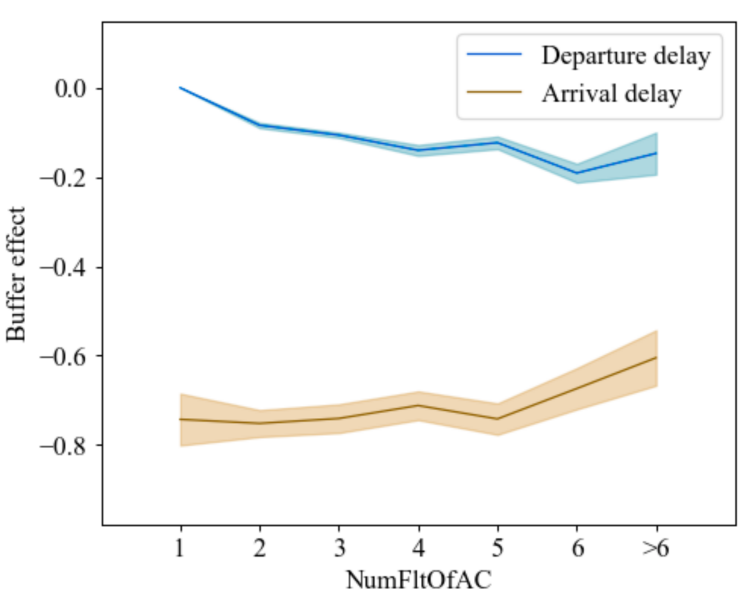

(b)

Figure 3. Delay propagation effect variation with OrdFltOfAC. (a) Previous delay effect against OrdFltOfAC. (b) Buffer effect against OrdFltOfAC.

\subsection{Sensitivity to the Number of Flight Legs}

In addition to the OrdOfFltAC affecting the flight-delay behavior, the NumOfFltAC will also impact flight on-time performance due to the delay propagation effect. The longer the NumOfFltAC, the more latter flights will be affected by the previous ones. Different from the flight order of an aircraft operating per day, this subsection focuses on the number of flight legs that the aircraft associated with the flight under study flies in a day, which is denoted as NumFltOfAC. Similar to the analysis of sensitivity to OrdFltOfAC, the flights of departure and arrival are also classified into seven groups based on NumFltOfAC: 1, 2, 3, 4, 5,6 , and $>6$. The distribution of the seven groups is shown in Figure 4. As Figure 4 shows, flights with a NumFltOfAC equaling four possess the largest part of the total flights both for departures and arrivals. In particular, the number of flights with a NumFltOfAC of four is twice that of flights with five NumFltOfACs, which is the second largest proportion. Flights associated with more than six legs a day only occupied less than two percent of the total flights.

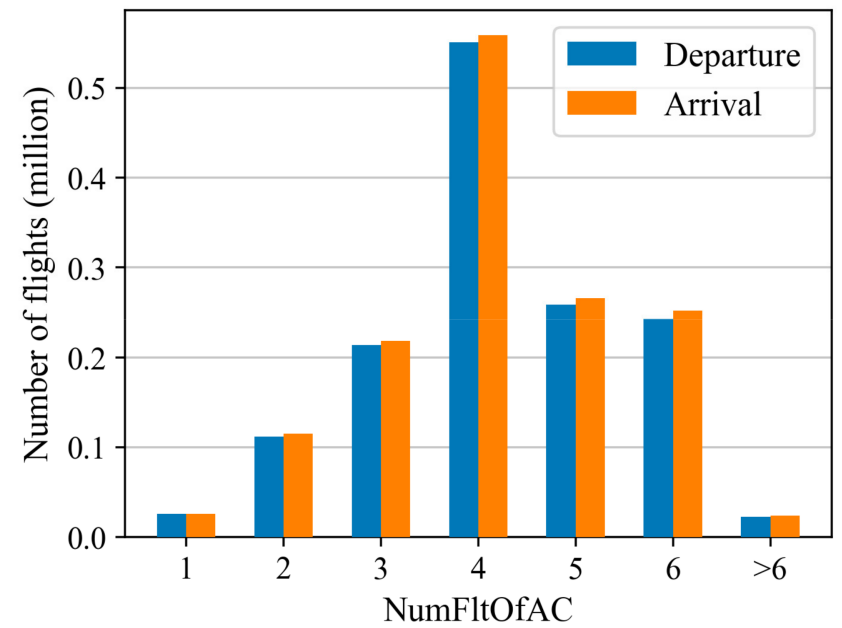

Figure 4. Distribution of the number of legs flown by aircraft in China.

Similar to the analysis of OrdFltOfAC, the estimation results are presented for each of the seven flight groups by NumFltOfAC for both departure and arrival delays in Table 5 . Again, all standard errors in the departure and arrival models are clustered by departure and arrival airports, respectively. Figure 5 plots the point estimates of the previous delay 
and buffer effect for different NumFltOfACs in the departure and arrival models along with 95\% confidence intervals. The coefficient variations of previous delay (Delay_PreDep and Delay_PreArr) are shown in Figure 5a. Delay_PreDep effect on arrival delays are larger overall than the Delay_PreArr effect on departure delays, which indicates that no matter how many flight legs an aircraft flies in a day. The propagation effect on arrival delays is larger than the effect on departure delays. The previous delay effect for arrival flights presents an increasing trend in general, except for a slight decrease when the NumFltOfAC is less than three. When an aircraft flies more than three flights in a day, the previous delay effect to flight delay tends to increase. For departure models, the influence of the previous delay presents a slight increasing trend, except when an aircraft flies more than six legs per day.

Table 5. Estimation results of flight delays for departure and arrival in different NumFltOfAC.

\begin{tabular}{|c|c|c|c|c|c|c|c|}
\hline Departure & 1 & 2 & 3 & 4 & 5 & 6 & $>6$ \\
\hline Delay_PreArr & 0 & $0.712^{* * *}$ & $0.640 * * *$ & $0.714^{* * *}$ & $0.692 * * *$ & $0.762 * * *$ & $0.710^{* * *}$ \\
\hline TT_dif & 0 & $-0.078 * * *$ & $-0.070 * * *$ & $-0.092 * * *$ & $-0.085 * * *$ & $-0.116^{* * *}$ & $-0.103^{* * *}$ \\
\hline CumDepDelay & $0.041^{* * *}$ & $0.046^{* * *}$ & $0.037^{* * *}$ & $0.031^{* * *}$ & $0.026^{* * *}$ & $0.023^{* * *}$ & $0.019^{* * *}$ \\
\hline Widebody & $18.589^{* * *}$ & $6.303^{* * *}$ & $4.767 * * *$ & 2.896 & $4.772 * * *$ & $6.724^{* *}$ & $-7.107^{* * *}$ \\
\hline HubDep & $-19.101^{* * *}$ & -11.233 & $-6.505^{* * *}$ & $-5.259 * * *$ & $-4.610 * * *$ & $-3.467 * * *$ & $-4.138 * *$ \\
\hline HubArr & $-13.569 * *$ & 0.077 & $1.940 * *$ & 0.759 & 0.531 & 0.31 & -0.768 \\
\hline Daytime & $-24.972 *$ & 1.386 & 0.155 & 0.911 & 0.051 & $2.126^{* * *}$ & 1.065 \\
\hline Weekday & 0.34 & 0.254 & 0.491 & $0.418^{* * *}$ & $0.496^{* * *}$ & 0.143 & 0.564 \\
\hline Sprg & 4.75 & 0.993 & 0.385 & -0.89 & -0.898 ** & $-1.039 * *$ & -0.399 \\
\hline Sumr & $15.269^{* * *}$ & $6.254^{* * *}$ & $3.756^{* * *}$ & $1.255 *$ & 1.055 & -0.373 & 0.116 \\
\hline Fall & $8.772 * * *$ & $3.771 * * *$ & $2.740 * * *$ & $1.778^{* * *}$ & $1.607^{* * *}$ & 0.158 & 0.665 \\
\hline ConvecWthrDep & -3.393 & 12.513 & $20.152 * * *$ & $17.319^{* * *}$ & $18.852^{* * *}$ & $14.230^{* * *}$ & $15.126^{* * *}$ \\
\hline ConvecWthrArr & $18.629^{* *}$ & $29.693^{* * *}$ & $27.843^{* * *}$ & $23.059 * * *$ & $18.928^{* * *}$ & $14.022 * * *$ & $11.846^{* * *}$ \\
\hline TempDepDif & 0.233 & 0.129 & 0.035 & -0.017 & -0.036 & -0.01 & 0.033 \\
\hline TempArrDif & 0.171 & 0.02 & -0.001 & 0.002 & 0.023 & -0.005 & 0.005 \\
\hline WdspDep & 0.999 & 0.741 ** & $0.609^{* * *}$ & $0.427^{* * *}$ & $0.242^{* * *}$ & $0.231^{* *}$ & 0.196 \\
\hline WdspArr & $1.804 * *$ & $0.942 * * *$ & $0.733 * * *$ & $0.447 * * *$ & $0.302 * * *$ & $0.133 * *$ & -0.159 \\
\hline VisDep & $-18.987 * * *$ & $-6.215^{* * *}$ & $-2.116^{* *}$ & -1.12 & -0.38 & 0.024 & $-1.537 * *$ \\
\hline VisArr & $-22.714 * * *$ & $-8.940 * * *$ & $-5.576^{* * *}$ & $-1.935 * * *$ & $-1.516^{* * *}$ & $-1.019^{* * *}$ & $-1.675 * *$ \\
\hline Cons & $103.344^{* * *}$ & $22.097^{* * *}$ & $9.622 * * *$ & $3.638^{* *}$ & 2.913 * & 0.23 & $7.087^{* * *}$ \\
\hline Num. Obs. & 26,144 & 111,656 & 213,746 & 550,703 & 258,848 & 243,084 & 22,507 \\
\hline$R^{2}$ & 0.080 & 0.322 & 0.332 & 0.445 & 0.46 & 0.544 & 0.511 \\
\hline Arrival & 1 & 2 & 3 & 4 & 5 & 6 & $>6$ \\
\hline Delay_PreDep & $0.778^{* * *}$ & $0.765^{* * *}$ & $0.751^{* * *}$ & $0.857^{* * *}$ & $0.884^{* * *}$ & $0.913^{* * *}$ & $0.983^{* * *}$ \\
\hline BT_dif & $-0.422 * *$ & $-0.805^{* * *}$ & $-0.778^{* * *}$ & $-0.739 * * *$ & $-0.778^{* * *}$ & $-0.760^{* * *}$ & $-0.920 * * *$ \\
\hline CumArrDelay & $0.011^{* * *}$ & $0.014^{* * *}$ & $0.012^{* * *}$ & $0.008^{* * *}$ & $0.006^{* * *}$ & $0.005^{* * *}$ & 0.002 \\
\hline Widebody & $-13.384^{* * *}$ & $-6.244^{* * *}$ & $-2.419 * * *$ & $-2.080 * * *$ & $1.928 *$ & 1.143 & -8.532 \\
\hline HubDep & -3.825 & $1.992 * * *$ & $-1.987^{* * *}$ & 0.228 & -0.974 * & -0.653 & -0.86 \\
\hline HubArr & $-4.318^{*}$ & -0.906 & $-1.915^{* *}$ & 0.027 & 0.19 & -0.235 & 0.253 \\
\hline Daytime & 0.168 & $3.714^{* * *}$ & $3.366^{* * *}$ & $2.357 * * *$ & $1.711^{* * *}$ & $1.587^{* * *}$ & $2.185^{* *}$ \\
\hline Weekday & 0.125 & 0.548 * & $0.688^{* * *}$ & $0.504^{* * *}$ & $0.623^{* * *}$ & $0.472 * * *$ & $0.479 *$ \\
\hline Sprg & $-4.083 * * *$ & $-1.789 * * *$ & $-2.545 * * *$ & $-1.058 * *$ & -0.457 & 0.321 & 0.86 \\
\hline Sumr & 2.039 & $1.142 * *$ & 0.075 & $-0.949 * *$ & -0.65 & $-1.409 * *$ & $-1.320 *$ \\
\hline Fall & 1.009 & -0.517 & -0.776 & $-1.375^{* *}$ & $-0.760 *$ & $-0.954 * *$ & -0.498 \\
\hline ConvecWthrDep & $18.410^{* * *}$ & $12.115^{* * *}$ & $11.308^{* * *}$ & $7.909 * * *$ & $8.623 * * *$ & $5.658 * * *$ & 1.559 \\
\hline ConvecWthrArr & $22.917 * * *$ & $15.803^{* * *}$ & $14.907^{* * *}$ & $12.579 * * *$ & $12.841^{* * *}$ & $10.333^{* * *}$ & $12.009 * * *$ \\
\hline TempDepDif & 0.161 * & $0.179 * * *$ & $0.144^{* * *}$ & $0.152 * * *$ & $0.082 * * *$ & 0.061 ** & -0.06 \\
\hline TempArrDif & -0.005 & 0.004 & 0.014 & -0.013 & -0.005 & -0.01 & $0.148^{* *}$ \\
\hline WdspDep & 0.341 & $0.215^{*}$ & 0.041 & -0.049 & -0.088 & -0.012 & -0.058 \\
\hline WdspArr & $1.209 * * *$ & $0.679 * * *$ & $0.460 * * *$ & $0.335 * * *$ & $0.361^{* * *}$ & $0.287^{* * *}$ & $0.185 *$ \\
\hline VisDep & $-4.253 * * *$ & $-3.559 * * *$ & $-2.864^{* * *}$ & $-1.624^{* * *}$ & $-1.381 * * *$ & $-1.153^{* * *}$ & 0.167 \\
\hline VisArr & $-3.802 * * *$ & $-2.146^{* * *}$ & $-1.542 * * *$ & $-1.194^{* * *}$ & -0.432 & -0.026 & 0.786 \\
\hline Cons & $33.857^{* * *}$ & $13.321^{* * *}$ & $12.186^{* * *}$ & $5.470^{* * *}$ & $4.119^{* * *}$ & $2.709^{* * *}$ & -2.052 \\
\hline Num. Obs. & 25,721 & 114,904 & 218,321 & 558,678 & 266,015 & 252,094 & 23,901 \\
\hline$R^{2}$ & 0.71 & 0.731 & 0.720 & 0.803 & 0.814 & 0.843 & 0.930 \\
\hline
\end{tabular}




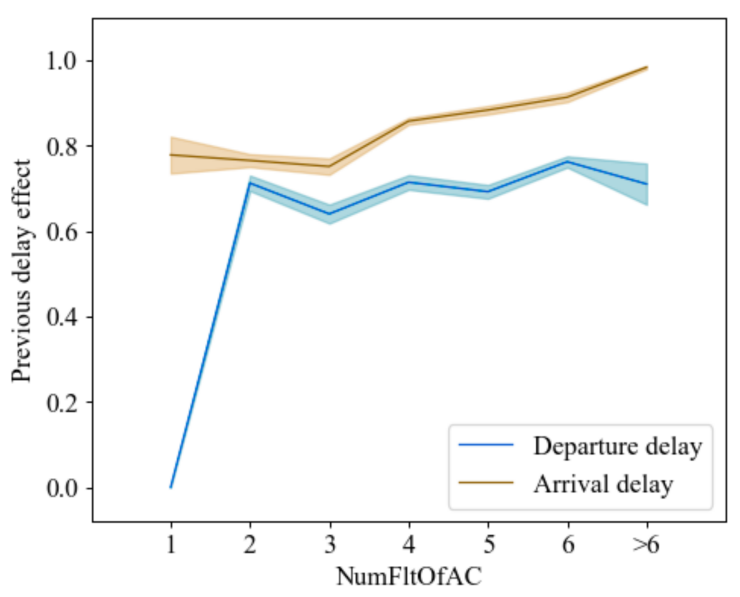

(a)

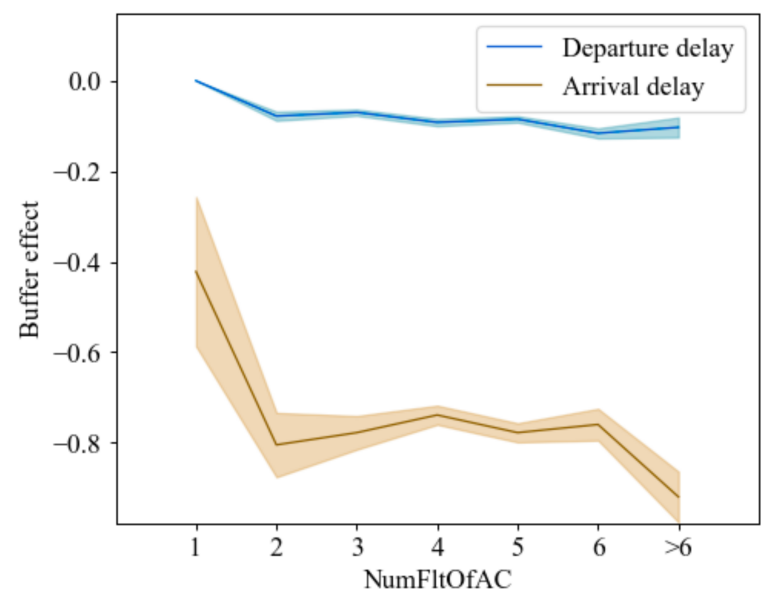

(b)

Figure 5. Delay propagation effect variation with NumFltOfAC. (a) Previous delay effect against NumFltOfAC. (b) Buffer effect against NumFltOfAC.

Figure $5 \mathrm{~b}$ shows that the point estimates of Buffer coefficients decrease (negative direction) with NumFltOfAC increasing, matching Hypothesis 4, both for departure and arrival models. The coefficients of block buffer (BT_dif) in arrival models is much smaller than that of turnaround buffer (TT_dif) in departure models, which indicates that flight airborne time absorbs more previous delays than aircraft on the ground no matter how many flight legs an aircraft flies in a day. In the arrival models, the BT_dif shows a decrease trend in general. The more flights an aircraft flies in a day, the longer the potential delay propagation chain is. A strong buffer effect can inhibit the delay propagation effect for flights with a large NumFltOfAC. Unlike in Section 6.1, the gap between TT_dif and BT_dif increases from 0.4 to 0.8 with the growth of NumFltOfAC.

\section{Conclusions}

Acknowledging the ever-growing demands of flights and the consequent serious flight delays, understanding the behaviors of flight delays is of critical importance. One contribution of this research is that econometric models are used to study the departure and arrival delays considering the operation-, time-, and weather-related variables in the main airports of China's air traffic network. Applying econometric models, the main results obtained show that arrival flights suffer more previous delays than departure flights, and the Buffer effects for arrival flights are also much greater than those of departure flights. Therefore, airlines can insert more buffer time in the airborne than on the ground, which is more efficient to mitigate the delay propagation effect. Convective weather is the most significant variable for both departure and arrival flights delays, and both are less impacted by convection weather in the original airport than that in the destination airport. However, the impact of convective weather on arrival delays is about half the impact of that of departure delays. Another major contribution of this paper is that sensitivity analysis on aircraft utilization is introduced so as to analyze the marginal effect of previous delays and buffer time varying with different NumFltOfAC and OrdFltOfAC. When airlines scheduling flights, this paper provides a perspective from aircraft utilization to improve on-time performance. For OrdFltOfAC, the later the flight order, the stronger the influence of the previous delay. Buffer effect shows the opposite situation, presenting an increased trend for departure flights and a reduction for arrivals. Therefore, for flights with a large OrdFltOfAC, inserting large block buffer time may not a useful strategy to decrease the arrival delay efficiently. For NumFltOfAC, the previous delay and buffer effect grow with the NumFltOfAC increasing. This result allows us to gauge the importance of flight order to delay propagation effect specific to individual flights when estimating departure and 
arrival delays. It indicates that providing more ground buffer is useful to reduce departure delay if an aircraft operates many flight legs per day.

The present approaches can be further extended in a few directions. First, a further direction is to provide a comparative analysis between China and the US, as both have the largest domestic air traffic industries. Analyzing the trend of the previous delay and buffer effect can provide a view of the delay propagation effect variation under different policies of both countries. Second, beside the aircraft utilization, more sensitivity analysis can be applied to describe the flight delays. One indicator is the complexity of en-route network. The more complex the en-route network, the more time aircraft spend cruising and on the ground, which makes flight delays propagate more easily in the network. Furthermore, it would be interesting to model flight delays by focusing not only on an airport network but also on an en-route network.

Author Contributions: Conceptualization, Z.Z. and W.W.; methodology, Z.Z. and W.W.; software, Z.Z.; validation, Z.Z.; formal analysis, Z.Z.; investigation, Z.Z.; resources, M.H.; data curation, Z.Z. and M.H.; writing-original draft preparation, Z.Z. and W.W.; writing—review and editing, Z.Z. and W.W.; visualization, Z.Z.; supervision, W.W.; project administration, Z.Z.; funding acquisition, M.H. and Z.Z. All authors have read and agreed to the published version of the manuscript.

Funding: This research was funded by National Natural Science Foundation of China (grant number 71971112) and China Scholarship Council (grant number 201806830077).

Institutional Review Board Statement: Not applicable.

Informed Consent Statement: Not applicable.

Data Availability Statement: Not Applicable.

Acknowledgments: We would like to extend our gratitude to Bo Zou, from Department of Civil, Materials and Environmental Engineering at University of Illinois at Chicago, whose constructive comments have helped us substantially improve the quality of the paper.

Conflicts of Interest: The authors declare no conflict of interest.

Appendix A. Major Hub Airports in China

\begin{tabular}{ll}
\hline Airlines & Hub Airports \\
\hline \multirow{3}{*}{ Air China } & Beijing Capital International Airport (PEK) \\
& Chengdu Shuangliu International Airport (CTU) \\
& Shanghai Pudong International Airport (PVG) \\
& Shanghai Hongqiao International Airport (SHA) \\
\hline \multirow{3}{*}{ Eastern Airlines } & Shanghai Pudong International Airport (PVG) \\
& Shanghai Hongqiao International Airport (SHA) \\
& Beijing Capital International Airport (PEK) \\
& Kunming Changshui International Airport \\
& Xi'an Xianyang International Airport (XIY) \\
& Guangzhou Baiyun International Airport (CAN) \\
Southern Airlines & Beijing Capital International Airport (PEK) \\
& Ürüqi Diwopu International Airport (URC) \\
& Chongqing Jiangbei International Airport (CKG) \\
& Shanghai Pudong International Airport (PVG) \\
& Haikou Meilan International Airport (HAK) \\
Hainan Airlines & Beijing Capital International Airport (PEK) \\
& Xi'an Xianyang International Airport (XIY) \\
\hline \multirow{3}{*}{ Xiamen Airlines } & Xiamen Gaoqi International Airport (XIA) \\
& Fuzhou Changle International Airport (FOC) \\
& Hangzhou Xiaoshan International Airport (HGH) \\
& Changsha Huanghua International Airport (CSX) \\
\hline \multirow{2}{*}{ Shanghai Airlines } & Shanghai Pudong International Airport (PVG) \\
\hline \multirow{2}{*}{ Shandong Airlines } & Shanghai Hongqiao International Airport (SHA) \\
& Jinan Yaoqiang International Airport (TNA) \\
& Qingdao Liuting International Airport (TAO) \\
& Xiamen Gaoqi International Airport (XIA) \\
\hline
\end{tabular}




\section{References}

1. World Bank. Air Transport, Passenger Carried. Available online: https://data.worldbank.org/indicator/IS.AIR.PSGR (accessed on 6 June 2021).

2. Joint Economic Committee (JEC). Your Flight Has Been Delayed Again: Flight Delays Cost Passengers, Airlines, and the US Economy Billions. Available online: https:/ /www.jec.senate.gov/public/index.cfm/democrats/2008/5/your-flight-has-beendelayed-again_1539 (accessed on 9 October 2020).

3. CAAC. Regulations on Flight On-Time Performance Management. Available online: http://www.gov.cn/gongbao/content/2016 / content_5115843.htm (accessed on 6 June 2021).

4. European Commission. Laying Down a Performance and Charging Scheme in the Single European Sky and Repealing Implementing Regulations (EU) No. 390/2013 and (EU) No. 391/2013. Available online: https:/ / eur-lex.europa.eu/legal-content/EN/ TXT/HTML/?uri=CELEX:32019R0317\&from=EN\#d1e32-32-1 (accessed on 20 July 2021).

5. Mayer, C.; Sinai, T. Why Do Airlines Systematically Schedule Their Flights to Arrive Late? The Wharton School, University of Pennsylvania: Philadelphia, PA, USA, 2003.

6. More, D.; Sharma, R. The turnaround time of an aircraft: A competitive weapon for an airline company. DECISION Off. J. Indian Inst. Manag. Calcutta 2014, 41, 489-497. [CrossRef]

7. Wong, J.T.; Tsai, S.C. A survival model for flight delay propagation. J. Air Transp. Manag. 2012, 23, 5-11. [CrossRef]

8. Boswell, S.B.; Evans, J.E. Analysis of Downstream Impacts of Air Traffic Delay; Lincoln Laboratory, Massachusetts Institute of Technology: Lexington, MA, USA, 1997.

9. Beatty, R.; Hsu, R.; Berry, L.; Rome, J. Preliminary evaluation of flight delay propagation through an airline schedule. Air Traffic Control Q. 1999, 7, 259-270. [CrossRef]

10. AhmadBeygi, S.; Cohn, A.; Guan, Y.; Belobaba, P. Analysis of the potential for delay propagation in passenger airline networks. J. Air Transp. Manag. 2008, 14, 221-236. [CrossRef]

11. Fricke, H.; Schultz, M. Delays Impacts onto Turnaround Performance Optimal Time Buffering for Minimizing Delay Propagation. 2009. Available online: http://www.atmseminar.org/seminarContent/seminar8/papers/p_153_AO.pdf (accessed on 10 November 2020).

12. Zheng, Z.; Wei, W.; Zou, B.; Hu, M. How Late Does Your Flight Depart? A Quantile Regression Approach for a Chinese Case Study. Sustainability 2020, 12, 10553. [CrossRef]

13. Kim, M.; Bae, J. Modeling the flight departure delay using survival analysis in South Korea. J. Air Transp. Manag. 2021, 91, 101996. [CrossRef]

14. Hao, L.; Hansen, M.; Zhang, Y.; Post, J. New York, New York: Two ways of estimating the delay impact of New York airports. Transp. Res. Part E Logist. Transp. Rev. 2014, 70, 245-260. [CrossRef]

15. Kafle, N.; Zou, B. Modeling flight delay propagation: A new analytical-econometric approach. Transp. Res. Part B Methodol. 2016, 93, 520-542. [CrossRef]

16. Pérez-Rodríguez, J.V.; Pérez-Sánchez, J.M.; Gómez-Déniz, E. Modelling the asymmetric probabilistic delay of aircraft arrival. J. Air Transp. Manag. 2017, 62, 90-98. [CrossRef]

17. Tan, X.; Jia, R.; Yan, J.; Wang, K.; Bian, L. An Exploratory analysis of flight delay propagation in China. J. Air Transp. Manag. 2021, 92, 102025. [CrossRef]

18. Baumgarten, P.; Malina, R.; Lange, A. The impact of hubbing concentration on flight delays within airline networks: An empirical analysis of the US domestic market. Transp. Res. Part E Logist. Transp. Rev. 2014, 66, 103-114. [CrossRef]

19. Abdel-Aty, M.; Lee, C.; Bai, Y.; Li, X.; Michalak, M. Detecting periodic patterns of arrival delay. J. Air Transp. Manag. 2007, 13, 355-361. [CrossRef]

20. Tu, Y.; Ball, M.O.; Jank, W.S. Estimating flight departure delay distributions-A statistical approach with long-term trend and short-term pattern. J. Am. Stat. Assoc. 2008, 103, 112-125. [CrossRef]

21. Lubbe, B.; Victor, C. Flight delays: Towards measuring the cost to corporations. J. Air Transp. Manag. 2012, 19, 9-12. [CrossRef]

22. Delgado, L.; Gurtner, G.; Bolić, T.; Castelli, L. Estimating economic severity of Air Traffic Flow Management regulations. Transp. Res. Part C Emerg. Technol. 2021, 125, 103054. [CrossRef]

23. Allan, S.S.; Gaddy, S.G.; Evans, J.E. Delay Causality and Reduction at the New York City Airports Using Terminal Weather Information Systems (No. ATC-291); Lincoln Laboratory, Massachusetts Institute of Technology: Cambridge, MA, USA, 2001.

24. Schaefer, L.; Millner, D. Flight delay propagation analysis with the detailed policy assessment tool. In Proceedings of the 2001 IEEE International Conference on Systems, Man and Cybernetics. e-Systems and e-Man for Cybernetics in Cyberspace (Cat.No.01CH37236), Tucson, AZ, USA, 7-10 October 2001; IEEE: Piscataway, NJ, USA, 2001; Volume 2, pp. $1299-1303$.

25. Golaszewski, R. Reforming air traffic control: An assessment from the American perspective. J. Air Transp. Manag. 2002, 8, 3-11. [CrossRef]

26. Chen, Y.H.; Lin, J.J. Determinants of flight delays at East Asian airports from an airport, route and network perspective. J. Air Transp. Manag. 2021, 94, 102064. [CrossRef]

27. Abdelghany, K.F.; Shah, S.S.; Raina, S.; Abdelghany, A.F. A model for projecting flight delays during irregular operation conditions. J. Air Transp. Manag. 2004, 10, 385-394. [CrossRef]

28. Xu, N.; Sherry, L.; Laskey, K.B. Multifactor model for predicting delays at US airports. Transp. Res. Rec. J. Transp. Res. Board 2008, 2052, 62-71. [CrossRef] 
29. Schultz, M.; Lorenz, S.; Schmitz, R.; Delgado, L. Weather impact on airport performance. Aerospace 2018, 5, 109. [CrossRef]

30. Klein, A.; Craun, C.; Lee, R.S. Airport delay prediction using weather-impacted traffic index (WITI) model. In Proceedings of the 29th Digital Avionics Systems Conference, Salt Lake City, UT, USA, 3-7 October 2010; p. 2-B.

31. Chatterji, G.; Sridhar, B. National airspace system delay estimation using weather weighted traffic counts. In AIAA Guidance, Navigation, and Control Conference and Exhibit; AIAA: San Francisco, CA, USA, 2005.

32. CAAC. Notice to Print and Distribute the On-Time Performance Statistical Methods for Civil Aviation Flights; CAAC: Beijing, China, 2012.

33. Britto, R.; Dresner, M.; Voltes, A. The impact of flight delays on passenger demand and societal welfare. Transport. Res. Part E Logist. Transp. Rev. 2012, 48, 460-469. [CrossRef]

34. Zou, B.; Hansen, M. Impact of operational performance on air carrier cost structure: Evidence from US airlines. Transp. Res. Part E Logist. Transp. Rev. 2012, 48, 1032-1048. [CrossRef]

35. FAA Density Altitude. FAA-P-8740-2 • AFS-8. 2008. Available online: https://www.faasafety.gov/files/gslac/library/ documents /2011/Aug/56396/FAA\%20P-8740-02\%20DensityAltitude\%5Bhi-res\%5D\%20branded.pdf (accessed on 4 August 2021).

36. Greene, W.H. Econometric Analysis, 7th ed.; Pearson Education: London, UK, 2012.

37. CAAC. Provisions on the Formulation and Implementation of the Minimum Standards for Aircraft Operations at an Airport. Available online: http:/ / www.gov.cn/gongbao/content/2002/content_61982.htm (accessed on 6 June 2021). 